\title{
Potencial do óleo essencial de noni no controle preventivo e curativo da antracnose da mangueira
}

\author{
Ana Clara Carneiro Fonseca ${ }^{a}$, Eliane Aparecida Rotilia ${ }^{a}$, Talita Pereira de Souza Ferreira ${ }^{a}$, \\ Dalmarcia de Souza Carlos Mourão ${ }^{a}$, Bruna Letícia Dias ${ }^{\mathrm{a}}$, Gabriella Rayssa Antunes da Silva Oliveira ${ }^{\text {, }}$ \\ Gil Rodrigues dos Santos ${ }^{a^{*}}$
}

\footnotetext{
${ }^{\text {a }}$ Universidade Federal do Tocantins (UFT), Brasil

*Autor correspondente (gilrsan@uft.edu.br)
}

\section{N F O}

\section{Keywords}

Morinda citrifolia $\mathrm{L}$. Colletotrichum gloeosporioides alternate control

\section{Palavras-chaves} Morinda citrifolia $\mathrm{L}$. Colletotrichum gloeosporioides controle alternativo

\begin{abstract}
A B S T R A C T
Noni essential oil potential in preventive and mango anthracnose control.

The use of essential oils has been used as an alternative in the control of diseases and pests can be a viable alternative. In the mango crop, anthracnose is considered the main disease because it causes damage in productivity and fruit quality. The objective of this work was to evaluate the use of noni essential oil in preventive and curative control of mango anthracnose. Phytopathogenic fungus was obtained from the isolation of characteristic lesions of mango plants. Essential oil was obtained from noni ripe fruits (Morinda citrifolia L.). In vitro bioassays were performed by testing different concentrations of essential oil. For the phytotoxicity test, five different concentrations of noni essential oil were used. The preventive control test was installed by applying through a manual spray. The dressing test was also performed. Noni essential oil has an inhibitory effect on mycelial anthracnose growth (C. gloeosporioides) in vitro on mango leaves. Symptoms of phytotoxicity were observed in mango leaves in noni oil doses greater than $2.0 \%$. The healing application of noni oil provided partial control of the disease. The healing application of noni oil provided partial control of the disease. In preventive form the use of noni oil is more efficient than curative in the control of anthracnose in mango plants. The application of fungicide provided preventive and curative control efficiency similar to noni oil, demonstrating that natural control can be a great choice of use in plant disease management.
\end{abstract}

\section{R E S U M O}

A utilização de óleos essenciais tem sido usado como uma alternativa no controle de doenças e pragas pode ser uma alternativa viável. Na cultura da manga, a antracnose é considerada a principal doença por causar prejuízos na produtividade e qualidade dos frutos. O objetivo desse trabalho foi avaliar a utilização de óleo essencial de noni no controle preventivo e curativo da antracnose da mangueira. $\mathrm{O}$ fungo fitopatogênico foi obtido a partir do isolamento de lesões características de plantas de manga. O óleo essencial foi obtido a partir de frutos maduros de noni (Morinda citrifolia L.). Os bioensaios in vitro foram realizados testando-se diferentes concentrações do óleo essencial. Para o teste de fitotoxicidade foi utilizado cinco concentrações diferentes de óleo essencial de noni. O teste de controle preventivo foi instalado aplicando-se por meio de um borrifador manual. Também foi realizado o teste curativo. O óleo essencial de noni têm efeito inibitório no crescimento micelial da antracnose (C. gloeosporioides) in vitro em folhas de manga. Os sintomas de fitotoxicidade foram observados nas folhas de manga em doses de óleo de noni maior que $2,0 \%$. A aplicação curativa do óleo de noni proporcionou controle parcial da doença. Na forma preventiva o uso de óleo de noni é mais eficiente que a curativa no controle da antracnose em plantas de manga. A aplicação do fungicida proporcionou eficiência de controle preventivo e curativo semelhante ao óleo de noni, demonstrando que o controle natural pode ser uma ótima opção de uso no manejo de doenças em plantas. 


\section{INTRODUÇÃO}

A planta de noni (Morinda citrifolia L.), pertence à família Rubiaceae, é um arbusto originário do sudoeste da Ásia que foi difundido pelo homem através da Índia e do Oceano Pacífico até as ilhas da Polinésia Francesa, onde se situa o Taiti. É uma planta que vem sendo utilizado pelos polinésios há cerca de 2.000 anos (Wang et al., 2002).

Há uma crescente preocupação da população em consumir alimentos saudáveis com uma produção associada à preservação do meio ambiente. A produção de alimentos com uma mínima degradação dos recursos naturais é uma exigência da sociedade, e nesse contexto destacam-se os alimentos portadores de selos que certificam a não utilização de agrotóxicos no processo produtivo (Silva et al., 2010).

Os óleos essenciais possuem alta atividade biológica e potencial para o controle de doenças de plantas, possuindo também baixa ou nenhuma toxidez aos mamíferos (Lima et al., 2016). Guimarães et al. (2011) ao estudarem o uso do óleo essencial de capim-limão (Cymbopogon citratus) comprovaram a inibição in vitro de Alternaria alternata. Aguiar et al. (2014) verificaram o uso de óleos essenciais de eucalipto (Corymbia citriodora) e capim-citronela (Cymbopogon nardus) no controle in vitro de Pyricularia grisea, Aspergillus spp. e Colletotrichum musae, obtendo resultados que demonstraram o potencial fungitóxico dos referidos óleos.

Dentre as técnicas de manejo sustentável, o uso de produtos naturais para o controle de patógenos tem se tornado uma realidade (Fonseca et al., 2015). Vários estudos demonstraram efeitos bioinseticidas, biofungicidas e bioherbicidas capazes de substituir pesticidas e reduzir os impactos ambientais (Ootani et al., 2013; Isman et al., 2011). Óleos essenciais de plantas têm sido em uso em todo o mundo, principalmente como repelentes de insetos (Isman, 2000). O efeito de compostos isolados extraídos de óleos essenciais de plantas atuam como fungicidas naturais inibindo a atividade fúngica e, um número significativo destes constituintes tem se mostrado eficaz (Pereira et al., 2006; 2018). Destes compostos destaca-se o cravo folha (Eugenea caryophyllus) contém alto teor de eugenol, substância que apresenta uma elevada atividade anestésica. O de hortelã pimenta (Mentha piperita) contém alto teor de mentol, mentona e mentofurano, substâncias que apresentam uma elevada atividade antibacteriana (Alves et al., 2010). O capim-limão (Cymbopogon citratus) contém alto teor de citral e limoneno, substâncias que apresentam uma elevada atividade fungicida. O manjericão (Ocimum basilicun) contém alto teor de linalol, substância que apresenta uma elevada atividade antisséptica (Pravuschi et al., 2010). A utilização de óleos essenciais tem tomado espaço cada vez maior, demostrandose como uma alternativa no controle de doenças e pragas (Ootani, 2013).

Apesar de vários estudos já terem sido realizados é importante à busca de mais conhecimentos visando a viabilidade técnica e econômica do uso de óleos essenciais, como ferramenta importante no combate aos fitopatógenos. Verificou-se escassez de trabalhos utilizando fungicidas botânicos no controle da antracnose da mangueira, uma importante doença da cultura que afeta diretamente a qualidade dos frutos. Desta forma, este trabalho objetivou avaliar a utilização de óleo essencial de noni no controle preventivo e curativo da antracnose da mangueira.

\section{MATERIAL E MÉTODOS}

O estudo foi desenvolvido em condições de laboratório e casa de vegetação, no Campus de Gurupi, na Universidade Federal do Tocantins.

\section{Obtenção e isolamento dos fitopatógenos}

O fungo fitopatogênico foi obtido a partir do isolamento de lesões características de plantas de manga, cultivar "Manga Rosa". Sua patogenicidade foi comprovada através dos postulados de Koch, conforme Silva et al. (2017).

\section{Extração do óleo essencial}

O óleo essencial foi obtido a partir de frutos maduros de noni (Morinda citrifolia L.), oriundos de plantas coletadas aleatoriamente do perímetro urbano no município de Gurupi/TO. Em seguida, foi extraído o óleo essencial dos frutos, pelo método de hidrodestilação por um período de duas horas. Em balão de fundo redondo com capacidade de 1000 $\mathrm{mL}$, foi depositado $0,02 \mathrm{~kg}$ de material para extração, acrescentando-se $500 \mathrm{~mL}$ de água destilada e em seguida acoplado em aparelho tipo Clevenger. Ao final do período de extração, os óleos essenciais foram coletados na forma de sobrenadante, armazenados em frascos âmbar, identificados e mantidos em geladeira a $4^{\circ} \mathrm{C}$ até o momento da implantação dos bioensaios (Seixas et al., 2012, adaptado).

\section{Testes in vitro}

Os bioensaios in vitro foram montados em placas de Petri (70 mm de diâmetro) testando-se diferentes concentrações do óleo essencial, composto majoritário e como testemunha. Foi utilizado delineamento experimental inteiramente casualizado 
em esquema bifatorial com três repetições e cinco épocas de avaliação $(2,4,6,8$ e 10 dias de incubação).

As concentrações de $0,25 \%, 0,5 \%, 1,0 \%, 1,5 \%$ e $2,0 \%$ dos óleos essenciais foram diluídas em solução de água esterilizada mais Tween 80 (1,0\%). Posteriormente, 100 microlitros das diferentes concentrações foram espalhadas na superfície do meio de cultura BDA comercial (Batata-Dextrose-Agar), com o auxílio de uma alça de Drigalsky, e em seguida, no centro de cada placa de Petri foi depositado um disco $(4 \mathrm{~mm})$ contendo micélio do fungo. As placas foram vedadas, identificadas e mantidas em câmara de incubação a $25^{\circ} \mathrm{C}$ por dez dias.

A avaliação foi feita pela mensuração do diâmetro médio das colônias em dois sentidos diametralmente opostos, com intervalos regulares de 48 horas. A partir dos valores obtidos do diâmetro médio dos fungos que foram isolados foi calculado o índice de velocidade de crescimento micelial (IVCM), adotando-se a fórmula descrita por Maia et al. (2011).

Sendo:

$$
I V C M=\frac{(\mathrm{D}-\mathrm{Da})}{N}
$$

$\mathrm{D}=$ diâmetro médio atual da colônia;

$\mathrm{Da}=$ diâmetro médio da colônia do dia anterior;

$\mathrm{N}=$ número de horas ou dias após a inoculação.

\section{Teste de Fitotoxicidade}

Cinco concentrações diferentes de óleo essencial de noni $(0,25 \% ; 0,5 \% ; 1,0 \% ; 1,5 \%$ e $2,0 \%$ v.v. $)$ foram usadas. Foi utilizado um controle, com aplicação de água destilada. Dez mililitros da solução de óleo essencial foram aplicados às plantas de manga (Mangifera indica L.), usando um pulverizador manual. As plantas foram mantidas a $25^{\circ} \mathrm{C} \pm 2^{\circ} \mathrm{C}$ por 48 horas. Posteriormente, foi realizada a avaliação da toxicidade nas plantas, utilizando-se uma escala de avaliação adaptada de (Dequech et al., 2008; Freitas et al., 2009; Cogliatti et al., 2011): 0\% = fitotoxicidade ausência; $1-25 \%=$ necrose leve das folhas ou planta de clorose leve; $26-50 \%=$ folhas moderadas necrose ou clorose de plantas moderadas; $51-75 \%$ = alta necrose de folhas ou alta clorose vegetal; $76-100 \%=$ murcha e planta seca.

\section{Controle preventivo e curativo}

Para as avaliações foram utilizadas plantas de manga da cultivar 'Manga-Rosa' que é uma das cultivares mais importantes do nordeste e muito conhecida no Brasil. As exigências edáficas para o cultivo da mangueira são solos de fertilidade e textura média, profundos e permeáveis é uma fruta de clima tropical e se adapta bem em regiões que apresentam as estações secas e chuvosas mais ou menos definidas. O solo ideal para o plantio da manga deve ter textura média, ser areno-argiloso (o que facilitará o desenvolvimento do sistema radicular), com boa drenagem, boa fertilidade e relevo plano ou pouco acidentado. Já a faixa de $\mathrm{pH}$ ideal para o cultivo de manga está em torno de 5,5 a 7,5. Chuva em excesso, em época inadequada, pode causar problemas à qualidade dos frutos, principalmente, se ocorrer durante a floração temperaturas médias entre 20 e $29^{\circ} \mathrm{C}$ são ideais para o seu cultivo (Araujo, 2004).

A partir dos testes de fitotoxicidade, foram estipuladas as concentrações de óleo essencial de noni e composto majoritário adotadas nos testes in vivo, no controle preventivo, sendo: $0,25 \%, 0,50 \%, 1 \%$ e $1,5 \%$, além de um tratamento com fungicida (Tiofanato Metílico) e testemunha com apenas água destilada. Em tubos de ensaio estéreis, adicionouse solução de água e Tween $80(1,0 \%)$, acrescentou-se a alíquota de óleo essencial para obtenção da concentração desejada, e em seguida, agitou-se até completa homogeneização. A solução de conídios dos fungos que foram isolados foi preparada, adicionando-se $20 \mathrm{~mL}$ de água destilada e esterilizada em placas de Petri. Em seguida, com o auxílio de um pincel de cerdas macias, realizou-se o desprendimento dos conídios e a solução obtida foi filtrada em gaze e efetuada a quantificação dos conídios em câmara de Neubauer, ajustando-se a concentração para $10^{6}$ conídios $\mathrm{mL}^{-1}$. O teste de controle preventivo foi instalado aplicando-se por meio de um borrifador manual, inicialmente, às diferentes concentrações nas plantas. Cerca de duas horas decorridas da aplicação, quando ocorreu a secagem da solução na superfície foliar, o patógeno foi inoculado nas folhas e em seguida as plantas foram mantidas em câmara úmida e escura por 48 horas. Posteriormente, as plantas foram colocadas em ambiente natural com temperatura variando de 26 a $34^{\circ} \mathrm{C} \pm 2^{\circ} \mathrm{C}$ para o desenvolvimento da doença. Decorridos 11 dias após a inoculação foram avaliadas a severidade da doença por meio da escala de notas adotada por Santos et al. (2005): $0=$ planta sadia; $1=$ menos de $1 \%$ da área foliar doente; $3=1$ a $5 \%$ da área foliar doente; $5=6$ a $25 \%$ da área foliar doente; $7=26$ a $50 \%$ da área foliar doente; $9=$ mais que $50 \%$ da área foliar doente.

Para avaliar o efeito curativo do óleo essencial, foi utilizado um delineamento inteiramente casualizado com 3 repetições e doses crescentes do óleo de $M$. citriofolia, sendo: $0,0,25 \% ; 0,50 \% ; 1,0 \%$, $1,5 \%$ e 2,0 . Como controle, foram utilizadas plantas pulverizadas com água (controle positivo) e plantas 
pulverizadas com fungicida Tiofanato Metílico (controle negativo). As plantas de manga foram inoculadas com $5 \mathrm{~mL}$ da solução de conídios $\left(10^{6}\right.$ conídios $\mathrm{mL}^{-1}$ ) de $C$. gloeosporioides. Em seguida, os vasos foram mantidos por $48 \mathrm{~h}$ fechado com saco plástico para fornecer uma câmara úmida. Após 48 $\mathrm{h}$ de inoculação, as plantas foram deixadas em local sombreado até o aparecimento da primeira folha com sintomas da doença. De cada tratamento, $5 \mathrm{~mL}$ das concentrações de óleo foram pulverizados sobre as plantas, após verificação do aparecimento da doença. A avaliação da severidade da doença foi realizada a cada três dias, sendo realizado um total de cinco avaliações após a aplicação das soluções de óleo. Com os resultados obtidos nas avaliações foi feita a área abaixo da curva de progresso da doença (AACPD).

O controle curativo e preventivo in vivo foi submetido à regressão linear. Os dados de área abaixo da curva de progresso da doença (AACPD) foram submetidos à análise de variância (ANOVA). As equações de regressão foram ajustadas usando o software Excel®.

\section{RESULTADOS E DISCUSSÃO}

Os postulados de Koch, comprovaram a patogenicidade do fungo Colletotrichum gloeosporioides às plantas de manga inoculadas (Figura 1).

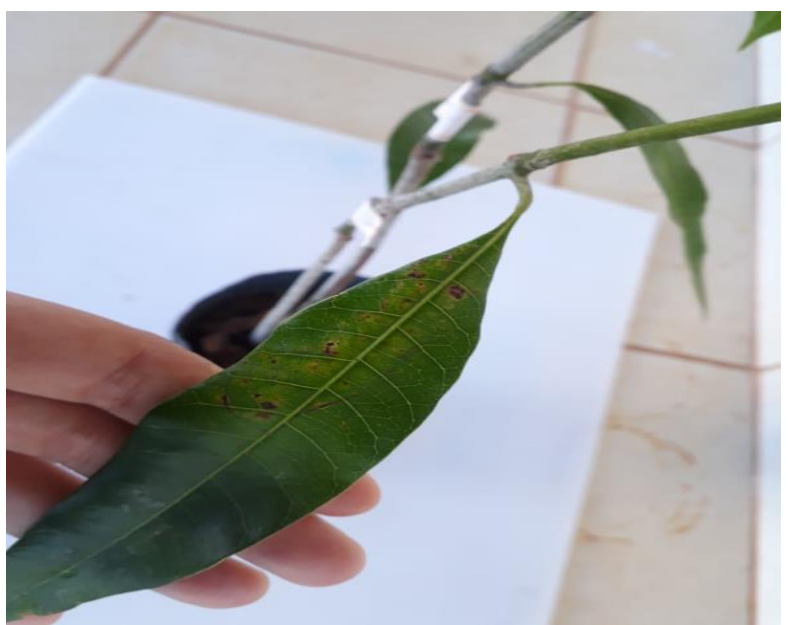

Figura 1 - Sintomas da antracnose em folhas de manga.

De acordo com os valores apresentados na Figura 2 a concentração de 2,0\% de óleo de $M$. citrifolia foi mais eficaz na inibição do cresceimento micelial de C. gloeosporioides, os valores foram maiores no sexto dia de incubação, já no décimo dia as diferenças observadas foram as menores do período avaliado.

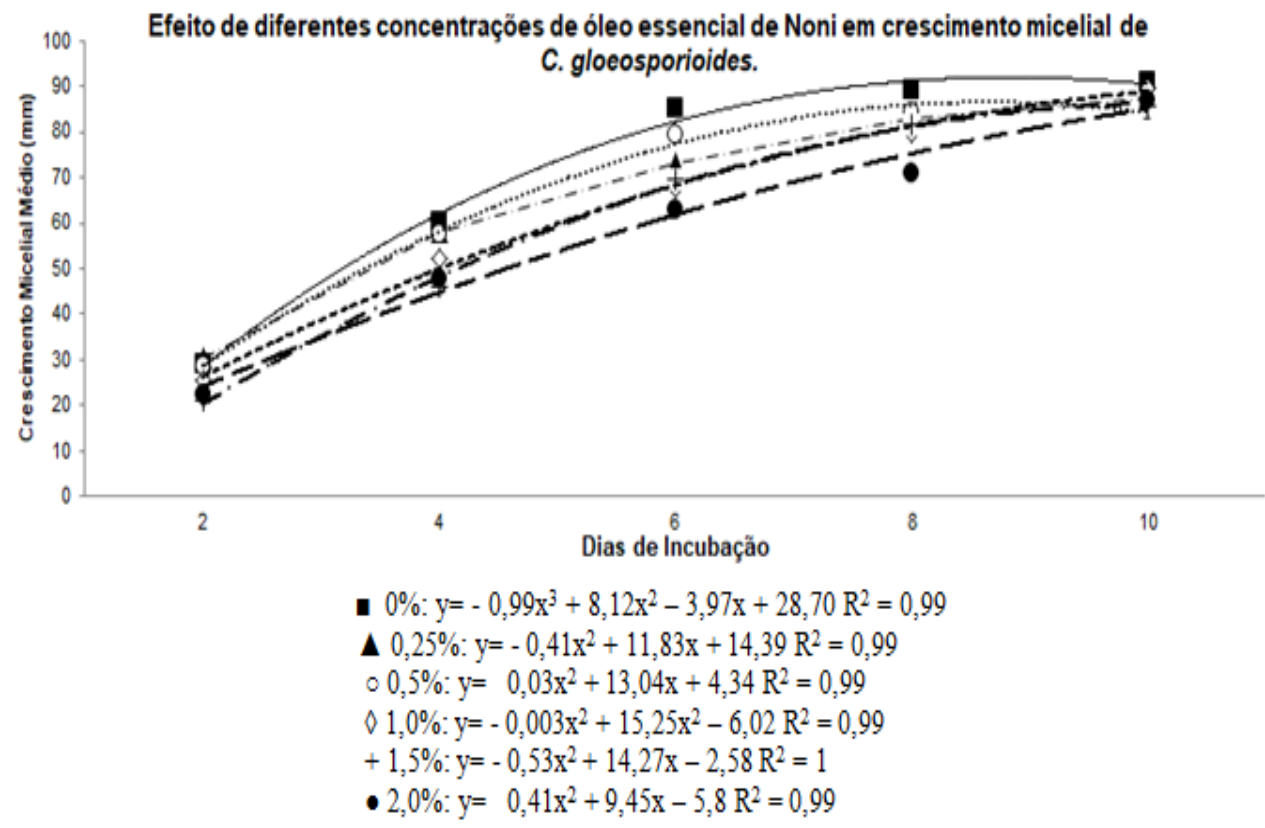

Figura 2 - Inibição do crescimento micelial in vitro de Colletotrichum gloeosporioides submetido a diferentes concentrações de óleo essencial de Morinda citrifolia.

Machado et al. (2013) avaliaram o crescimento micelial de C. gloeosporioides, causador de antracnose em plantas de manga com diferentes óleos, que apresentou efeito significativo a partir do $3^{\circ}$ dia de incubação do fungo $(\mathrm{P}<0,05)$. Os resultados mostraram maior inibição do crescimento micelial no $5^{\circ}$ dia em relação aos controles: água destilada esterilizada ou Tween 80 .

A partir do $8^{\circ}$ dia de incubação a eficácia do óleo de noni foi reduzida, isso pode ser explicado devido 
à provável volatilidade dos compostos presentes no óleo, ou seja, capacidade que óleo possui de evaporação. Em todas as concentrações ao longo do tempo foi possível ajustar um modelo polinominal de segundo grau, onde os ponto máximos atingidos nas concentrações $0,0 \mu \mathrm{L}^{-1} \mathrm{~mL}, 25 \mu \mathrm{L}^{-1} \mathrm{~mL}, 50 \mu \mathrm{L}^{-}$ ${ }^{1} \mathrm{~mL}, 100 \mu \mathrm{L}^{-1} \mathrm{~mL}, 150 \mu \mathrm{L}^{-1} \mathrm{~mL}$ e $200 \mu \mathrm{L}^{-1} \mathrm{~mL}$ foram: $3,73 \mu \mathrm{L}^{-1} \mathrm{~mL} 14,2 \mu \mathrm{L}^{-1} \mathrm{~mL}, 3,83 \mu \mathrm{L}^{-1} \mathrm{~mL}, 5,7$ $\mu \mathrm{L}^{-1} \mathrm{~mL}, 0,48 \mu \mathrm{L}^{-1} \mathrm{~mL}$ respectivamente. Nas demais concentrações de $1,5 \%$ e 2,0 não obteve-se ponto máximo, pois não estão inseridos no intervalo estudado.

\section{Teste in vivo}

Foi observada toxicidade a partir da concentração de 2,0\% de óleo essencial de Morinda citrifolia em plantas de manga, apresentando sintomas de necrose e clorose leve variando de $1 \%$ a $25 \%$ do limbo foliar (Tabela 1).

Com base nos resultados obtidos nos teste in vitro e fitotoxicidade foram realizados os testes de controle preventivo e curativo. Ao analisar a AACPD (Figura 3) percebe-se que o óleo essencial de noni (Morinda citriofolia L.) possui pouca efetividade no controle curativo da antracnose, provavelmente devido não apresentar boa capacidade de translocação no interior dos tecidos infectados da planta. No entanto, sua aplicação em plantas de manga manteve estável o nível de severidade da doença, impedindo que ela evoluísse nas plantas causando mais danos. No teste curativo as concentrações de $0,25 \%, 0,50 \%$ e $1,0 \%$ reduziram até metade da doença (AACPD) quando comparado com a testemunha. Na menor concentração $0,25 \%$ o teste curativo apresentou efeito semelhante às concentrações do óleo no teste preventivo e esse efeito foi maior que as outras concentrações (Figura 3 ). Neste trabalho óleo apresentou maior ou igual eficiência quando comparado ao fungicida. Dessa forma, houve o controle parcial da doença, atrasando a evolução da mesma. Osorio et al. (2018) comprovaram que o controle da ferrugem na Teca obtido pela aplicação do óleo essencial, de noni foi parcial, possivelmente devido à presença de compostos tóxicos em grande quantidade nos óleos essenciais.

Tabela 1 - Fitotoxicidade de óleo essencial de $M o$ rinda citrifolia em função da aplicação de concentrações crescentes em plantas de manga

\begin{tabular}{c|c}
\hline Tratamentos & Sintomas de fitotoxidade \\
\hline 0 & $0 \%$ \\
0,25 & $0 \%$ \\
0,5 & $0 \%$ \\
1,0 & $0 \%$ \\
1,5 & $0 \%$ \\
2,0 & $1 \%-25 \%=$ necrose e clo- \\
& rose leve das folhas \\
\hline
\end{tabular}

As concentrações de óleo para efeito preventivo foram muito eficazes, semelhantes ao fungicida, sendo que todas as concentrações aplicadas não apresentaram sintomas de doenças nas plantas (Figura 3).

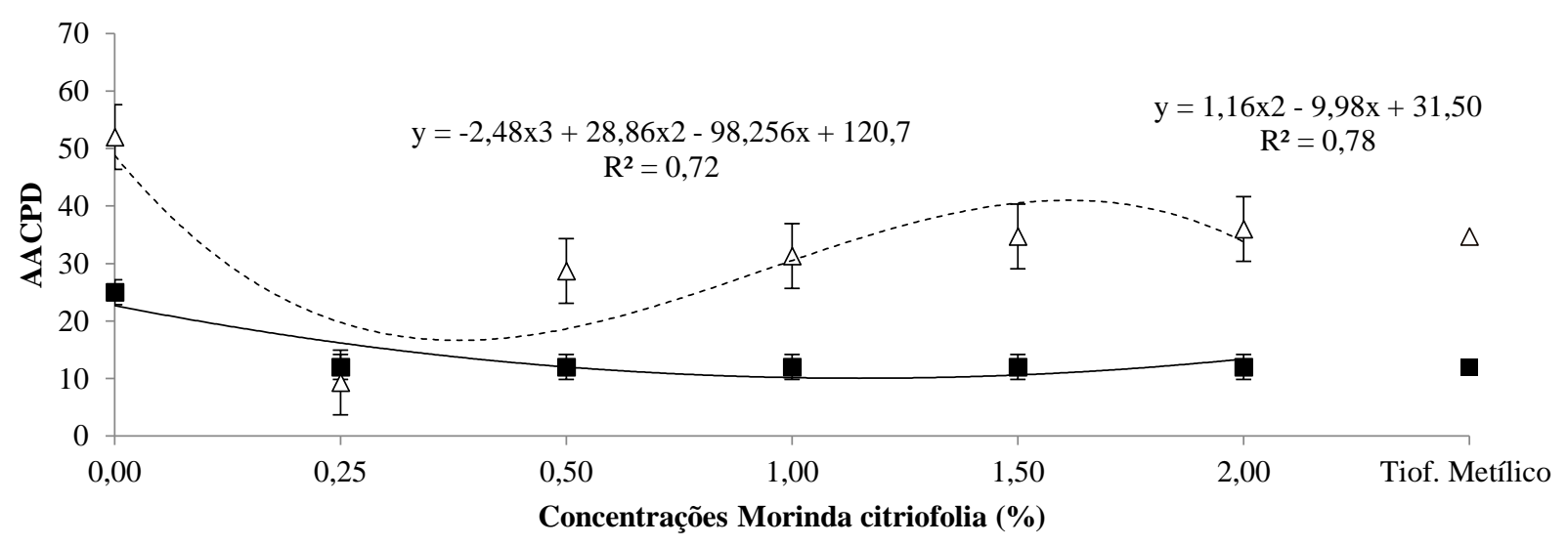

- PREVENTIVO $\triangle$ CURATIVO $\rightarrow$-Tiof. Metílico $\triangle$ Tiof. Metílico

Figura 3 - Área abaixo da curva de progresso (AACPD) para controle preventivo e curativo da antracnose da manga com óleo essencial de noni. Controle positivo com água $\left(0=0,0 \mu \mathrm{L}^{-1} \mathrm{~mL}\right)$ e controle negativo com o fungicida Tiofanato Metílico (Tiof. Metilico); concentrações de óleo de noni $\left(0,25 \%=25 \mu \mathrm{L}^{-1} \mathrm{~mL} ; 0,50 \%=\right.$ $50 \mu \mathrm{L}^{-1} \mathrm{~mL} ; 1,0 \%=100 \mu \mathrm{L}^{-1} \mathrm{~mL} ; 1,50 \%=150 \mu \mathrm{L}^{-1} \mathrm{~mL}$ e $\left.2,00 \%=200 \mu \mathrm{L}^{-1} \mathrm{~mL}\right)$. 
De acordo com o grau de fitotoxicidade causado pelo óleo essencial de noni, nas concentrações testadas, foi selecionada a concentração de máxima de $2,0 \%$ a ser utilizada nos testes de controle preventivo e curativo da antracnose em plantas de manga.

O óleo de noni obteve melhor eficiência no controle preventivo quando comparado ao curativo. Estes resultados são semelhantes aos encontrados por Osorio et al. (2018) que apontam resultados semelhantes para o óleo de noni. Sahoo et al. (2012) observaram potencial fungitóxico do extrato de folhas de noni sobre as espécies fúngicas Aspergillus niger, Aspergillus fumigatus, Rhizopus oryzae, Helminthosporium sp. Curvularia sp. e Sclerotium sp., consideradas importantes fitopatógenos em diversas culturas.

\section{CONCLUSÕES}

O óleo essencial de noni têm efeito inibitório no crescimento micelial da antracnose $(C$. gloeosporioides) in vitro em folhas de manga.

Os sintomas de fitotoxicidade foram observados nas folhas de manga em doses de óleo de noni maior que $2,0 \%$.

A aplicação curativa do óleo de noni proporcionou controle parcial da doença.

A aplicação preventiva do óleo de noni é mais eficiente que a curativa para o controle de antracnose em plantas de manga.

A aplicação do fungicida proporcionou eficiência de controle preventivo e curativo semelhante ao óleo de noni, demonstrando que o controle natural pode ser uma ótima opção de uso no manejo de doenças em plantas.

\section{REFERÊNCIAS BIBLIOGRÁFICAS}

ALVES, L.A.; FREIRES, I.A.; CASTRO, R.D. Efeito antibacteriano de óleos essenciais sobre bactérias formadoras do biofilme dentário. Revista Brasileira de Ciências da Saúde, v. 14, n. 2, p. 57-62, 2010.

https://doi.org/10.4034/RBCS.2010.14.02.07

ARAÚJO, J.L.P. Mercado e comercialização da manga. In: Mouco, MAC. Cultivo da Mangueira. Petrolina: Embrapa Semi-Árido, Sistemas de Produção, 2. Versão eletrônica, 2010.

AGUIAR, R.W.S.; OOTANI, M.A.; ASCENCIO, S.D.; FERREIRA, T.P.S.; SANTOS, M.M.; SANTOS, G.R. Fumigant antifungal activity of Corymbia citriodora and Cymbopogon nardus essential oils and citronella against three fungal species. The Science World Journal, v. 2014, p. 1-8, 2014. https://dx.doi.org/10.1155/2014/492138

COGLIATTI, M.; JUAN, V.F.; BONGIORNO, F.; DALLA VALLE, H.; ROGERS, W.J. Control of grassy weeds in anual canarygrass. Crop Protection, v.30, p.125-129, 2011. http://dx.doi.org/10.1016/j.cropro.2010.11.002
DEQUECH, S.T.B.; RIBEIRO, L.P.; SAUSEN, C.D.; EGEWARTH, R.; KRUSE, N.D. Fitotoxicidade causada por inseticidas botânicos em feijão-de-vagem (Phaseolus vulgaris L.) cultivado em estufa plástica. Revista da FZVA, v.15, n.1, p.71-80, 2008.

FONSECA, M.C.M.; LEHNER, M.D.S.; GONÇALVES, M.G.; JÚNIOR, P.; SILVA, A.F.; BONFIM, F.P.G.; PRADO, A.L. Potencial de óleos essenciais de plantas medicinais no controle de fitopatógenos. Revista Brasileira de Plantas Medicinais, v.17, n.1, p.45-50, 2015. https://dx.doi.org/10.1590/1983-084X/12_170.

FREITAS, S,P.; MOREIRA, J.G.; FREITAS, I.L.J.; FREITAS JÚNIOR, S.P.; AMARAL JÚNIOR, A,T.; SILVA, V.Q.R. Fitotoxicidade de herbicidas a diferentes cultivares de milho-pipoca. Planta Daninha, v.27, p.1095-1103, 2009. http://dx.doi.org/10.1590/S0100-83582009000500023.

GUIMARÃES, L.G.L.; CARDOSO, .MG.; SOUSA, P.E.; ANDRADE, J.; VIEIRA, S.S. Atividades antioxidante e fungitóxica do óleo essencial de capim-limão e do citral. Revista Ciência Agronômica, v.42, n.2, p.464-472, 2011.

ISMAN, M.B.; MIRESMAILLI, S.; MACHIAL, C. Commercial opportunities for pesticides based on plant essential oils in agriculture, industry and consumer products. Phytochemistry Reviews, v.10, n.2, p.197-204, 2011. https://doi.org/10.1007/s11101-010-9170-4.

ISMAN, M.B. Plant essential oils for pest and disease management. Crop Protection, v.19, n.8, p.603-608, 2000.

LIMA, A.F.; CRUZ, T.P.; FERREIRA, M.M.; COSTA, A.V.; JUNIOR, W.C.J.; MORAES, W.B.; QUEIROZ, V.T.; ALVES, F.R. Avaliação do efeito fungicida de óleos essenciais sobre a produção de esporos do fungo Fusarium solani. Revista Univap, v.22, n.40, p.802, 2016. http://dx.doi.org/10.18066/revistaunivap.v22i40.1619.

MACHADO, R.M.A.; DIAS, V.M. de SOUZA, C.L.M.; da SILVA, L.B.; FREIRE, M.D.G.M. Avaliação de óleos essenciais sobre o crescimento in vitro do fungo Colletotrichum gloeosporioides. Biológicas \& Saúde, v. 3, n. 8, p. 65-67, 2013. https://doi.org/10.25242/8868382013147.

MAIA, F.G.M.; ARMESTO, C.; ZANCAN, W.L.A.; MAIA, J.B.; ABREU, M.S. de. Efeito da temperatura no crescimento micelial, produção e germinação de conídios de Colletotrichum spp. isolados de mangueira com sintomas de antracnose. Bioscience Journal, v.27, n.2, p.205-210. 2011.

OOTANI, M,A.; AGUIAR, R.W.; RAMOS, A.C.; BRITO, D.R.; SILVA, J.B.D.; CAJAZEIRA, J.P. Use of essential oils in agriculture. Journal of Biotechnology and Biodiversity, v.4, n.2, p.162-175, 2013.

OSORIO, P.R.A.; LEÃO, E.U.; VELOSO, RA.; MOURÃO DSC.; SANTOS, G.D.O.S. Essential Oils for Alternative Teak Rust Control. Floresta e Ambiente. [online], v.25, n.2, p.6-9, 2018. https://dx.doi.org/10.1590/21798087.039116

PEREIRA, M.C.; VILELA, G.R.; COSTA, L.M.A.S., SILVA, R.F.; FERNANDES, A.F.; FONSECA, E.W.N.; PICCOLI, R.H. Inibição do desenvolvimento fúngico através da utilização de óleos essenciais de condimentos. Ciências e Agrotecnologias, v.30, n.4, p.731-738, 2006. https://dx.doi.org/10.1590/S1413-70542006000400020. 
PEREIRA, M.N.; CONCEIÇÃO, R.B. da; CRUZ, J.C.S.; ANDRADE, M.C.N. de. Efeito de óleos essenciais sobre o fungo Thielaviopsis paradoxa. Ambiência, v.14, n.3, 2018. https://dx.doi.org/10.5935/ambiencia.2018.03.0

PRAVUSCHI, P.R.; MARQUES, P.A.A.; RIGOLIN, B.H.M.; SANTOS, A.C.P. Efeito de diferentes lâminas de irrigação na produção de óleo essencial do manjericão (Ocimum basilicum L.). Acta Scientiarium Agronomy, v.32, n.4, p.687-693, 2010. DOI:

https://dx.doi.org/10.4025/actasciagron.v32i4.3160

SAHOO, K.; DHAL, N.K.; SAHOO, S.L.; LENKA, S.S. Comparative phytochemical and antimicrobial study of Morinda pubescens sm. and Morinda citrifolia L. International Journal of Pharmacy and Pharmaceutical Sciences, v.4, n.3, 425-429p., 2012.

SANTOS, G.R.; CAFÉ-FILHO, A.C.; SABOYA, L.M.F. Controle químico do crestamento gomoso do caule em melancia. Fitopatologia Brasileira, v.30, n.2, p.155-163, 2005. http://dx.doi.org/10.1590/S0100-41582005000200009

SEIXAS, P.T.L.; CASTRO, H.G.; CARDOSO, D.P.; CHAGAS, A.F JR.; NASCIMENTO, I.R. Bioactivity of essential oils on the fungus Didymella bryoniae of the cucumber culture. Brazilian Journal of Applied Technology for Agricultural Science, v. 5, n. 3, p. 61-66, 2012.

https://doi.org/10.5777/paet.v5i3.1978

SILVA, J,C,E.; MOURÃO, D.S.C.; LIMA, F.S.O.; SARMENTO, R.A.; DALCIN, M.S.; AGUIAR, R.W.S.; SANTOS, G.R. The efficiency of noni (Morinda citrifolia L.) essential oil on the control of leaf spot caused by Exserohilum turcicum in maize culture. Medicines, v. 4, n. 60; p.110, 2017. https://doi.org/10.3390/medicines 4030060

SILVA, M.B.; MORANDI, M.A.B.; PAULA JÚNIOR, T.J.; VENZO, M.; FONSECA, M.C.M. Uso de princípios bioativos de plantas no controle de fitopatógenos e pragas. Informe Agropecuário, v.31, n.255, p.70-77, 2010.

WANG, M.Y.; WEST, B.J.; JENSEN, C.J.; NOWICKI, D. Morinda citrifolia (Noni): A literature review and recent advances in Noni research. Acta Pharmacologica Sinica, v.23, n.12, p.1127-1141, 2000. 